\title{
Advantages of Orthogonal Folding of Single Polymer Chains to Soft Nanoparticles
}

\author{
Angel J. Moreno, ${ }^{1,2, *}$ Federica Lo Verso, ${ }^{2}$ Ana Sánchez-Sánchez, ${ }^{1,2}$ \\ Arantxa Arbe, ${ }^{1,2}$ Juan Colmenero, ${ }^{1,2,3,4}$ and José A. Pomposo ${ }^{1,2,4,5}$ \\ ${ }^{1}$ Centro de Física de Materiales (CSIC, UPV/EHU), \\ Paseo Manuel de Lardizabal 5, E-20018 San Sebastián, Spain. \\ ${ }^{2}$ Materials Physics Center MPC, Paseo Manuel de Lardizabal 5, E-20018 San Sebastián, Spain. \\ ${ }^{3}$ Donostia International Physics Center, Paseo Manuel de Lardizabal 4, E-20018 San Sebastián, Spain. \\ ${ }^{4}$ Departamento de Física de Materiales, Universidad del País Vasco (UPV/EHU), \\ Apartado 1072, E-20080 San Sebastián, Spain. \\ ${ }^{5}$ IKERBASQUE - Basque Foundation for Science, Alameda de Urquijo 36, E-48011 Bilbao, Spain
}

(Dated: October 10, 2013)

\begin{abstract}
We investigate, by means of computer simulations, the formation of soft nanoparticles by irreversible intramolecular cross-linking of homofunctional polymer precursors in good solvent. Simulations reveal that the early and intermediate stages of the cross-linking process are dominated by bonding at short contour distances. Because of the initial self-avoiding character of the precursor, bonding at long contour distances, which is the efficient mechanism for global compactation, is a rare event that essentially occurs in the late stage of cross-linking. Thus, irreversible crosslinking of precursors with identical molecular weight and linker fraction produces both compact and sparse objects. This is confirmed by a detailed analysis of the size and shape distribution of the fully cross-linked nanoparticles. We also investigate intramolecular cross-linking of heterofunctional polymers with two species of orthogonal linkers, bonding between distinct species being forbidden. It is found that simultaneous cross-linking of both species and sequential cross-linking (activation of one species after full cross-linking of the other) lead to the same structural properties for the resulting nanoparticles. The heterofunctional nanoparticles are on average smaller and more spherical than the homofunctional counterparts, though still a significant fraction of sparse objects is found. The simulation results are compared with results from SEC/MALLS and SAXS experiments in real polymeric nanoparticles.
\end{abstract}

\section{INTRODUCTION}

Efficient folding of single polymer chains is a topic of current interest due, mainly, to the predicted emergence in the near future of single-chain technologies (e.g., single-chain nanodevices) [1] and the attracting possibility to mimic the structure and functionality of natural biomacromolecules (e.g., enzymes, drug delivery vehicles, catalysts) by means of artificial single-chain nano-objects $[2,3]$. Indeed unimolecular polymeric nanoparticles have already been considered in several applications as, e.g., promising elastomeric polymers, rheology agents, sensors or smart gels [4-8].

Current folding protocols for synthetic (co)polymers rely on the use of intrachain cross-linking chemistries involving covalent $[2,5,6,9-26]$, non-covalent [27-33] or dynamic covalent bonds $[34,35]$. Additionally, techniques like intrachain homocoupling, intrachain heterocoupling or cross-linking induced collapse are available to promote the intramolecular folding/collapse of individual polymer chains to single-chain nanoparticles (SCNPs) [3]. However, the current folding degree in SCNPs is still far from that observed in globular proteins even by using highly-efficient 'click' chemistry techniques. In this sense, recent small-angle neutron scattering (SANS) $[2,26,36]$

${ }^{*}$ Corresponding author: wabmosea@ehu.es and small-angle X-ray scattering (SAXS) [37, 38] measurements of SCNPs in solution have shown form factors more closely related to those of disordered proteins (crumpled coils) than those expected for globular, strongly folded nano-objects. Even if globular morphologies have been observed in the dry state by Atomic Force Microscopy (AFM) [12, 14, 26, 28, 37] and Transmission Electron Microscopy (TEM) [2, 13, 26, 29, 31, 35, 38], SANS and SAXS data exclude the existence of such compact morphologies in solution at good solvent conditions. The origin of this kind of coil-to-globule transition upon solvent removal and nanoparticle deposition onto a substrate is still not well-understood, but its control is of paramount importance for the future development of single-chain biosensors, bioinspired unimolecular catalysts or responsive single-chain nanocarriers.

The lack of folding control during SCNP formation may be attributed to several factors including, among others, the presence of random monomer sequences in the single-chain copolymer precursor chains, unbalanced precursor-solvent interactions, or the inherent size dispersity of precursors synthesized through controlled radical polymerization techniques, when compared to perfectly monodisperse biomacromolecules [1]. Very recently, two different attempts have been carried out to improve the folding degree of SCNPs by using heterofunctional polymers and orthogonal cross-linking chemistries [36, 39]. However, the possibility of extracting general conclusions about the actual degree of folding improvement from these works is severely limited, due to the lack of compar- 
ison with the homofunctional (control) systems [36] and the use of precursors of relatively low molecular weight [39].

In this article we aim to draw a clear and general picture of the advantages of folding single polymer chains containing heterofunctional $(\mathrm{A}, \mathrm{B})$ reactive groups to soft nanoparticles via orthogonal intrachain $(\mathrm{A}+\mathrm{B})$ crosslinking techniques. With these ideas in mind, we perform molecular dynamics (MD) simulations of a generic bead-spring model for $\mathrm{A}+\mathrm{B}$ cross-linked nanoparticles and their exactly equivalent homofunctional counterparts (i.e., A or B cross-linked nanoparticles). Furthermore, we compare the MD simulation results with experimental results by size exclusion chromatography/multi-angle laser light scattering (SEC/MALLS) and SAXS measurements on real single-chain nanoparticles.

We find that the cross-linking process produces both compact and sparse topologies for the soft nanoparticles even if these have identical molecular weight and fraction of cross-linkers. This qualitative observation is consistent with the few simulation works reported for intramolecularly cross-linked nanoparticles [40-42]. However a detailed quantitative characterization of the structural properties of such soft nanoparticles is still lacking. In this article we perform extensive simulations to determine their scaling behavior, shape parameters (asphericity and prolateness) and internal structure (Euler characteristic). As an additional novel result, we discuss differences between such properties for homofunctional and heterofunctional nanoparticles. We find that the heterofunctional nanoparticles are, on average, smaller and more spherical than their homofunctional counterparts. Furthermore, we find that the simultaneous and sequentail cross-linking routes lead to the same structural properties of the obtained heterofunctional nanoparticles. The simulation results are consistent with the experimental results obtained by SEC/MALLS and SAXS.

The remaining of the paper is organized as follows: In Section II we describe the nanoparticle synthesis and the SEC/MALLS and SAXS characterization techniques. In Section III we describe the simulated model and give MD details. In Section IV we discuss simulation results for the scaling properties and the shape and internal structure of the obtained soft nanoparticles. Moreover we compare results for the heterofunctional nanoparticles obtained by both simultaneous and sequential crosslinking. In Section V, we compare the results from SEC/MALLS and SAXS experiments with the predictions from the MD simulations. Finally, Section VI summarizes the main conclusions of this work.

\section{EXPERIMENTAL DETAILS}

\section{A. Nanoparticle synthesis}

To test the main results of the MD simulations, and to show the advantages (smaller size, more spherical ob- jects) of folding single polymer chains containing heterofunctional reactive groups to soft nanoparticles, we selected a poly(methyl methacrylate) precursor containing $35 \mathrm{~mol} \%$ of reactive $\beta$-ketoester functional groups, and two kind of cross-linking techniques: Michael addition [43] using bifunctional acrylates, and metal complexation using divalent metal $\left(\mathrm{Cu}^{2+}\right)$ ions. Unfortunately, both cross-linking techniques cannot be activated simultaneously due to the interference of one technique over the other. In this sense, we found that the basic catalyst used in the Michael reaction induced the precipitation of the $\mathrm{Cu}^{2+}$ ions in solution. However, we found that they can be efficiently carried out in a sequential manner that, according to MD simulation results (see below), leads to the same nanoparticle conformations than by performing the simultaneous cross-linking process. Hence, we controlled the first cross-linking reaction (Michael addition) in such a way that half of the $\beta$-ketoester groups of the polymer precursor were consumed (i.e., half of the $\beta$-ketoester groups take formally the role of typeA groups). After that, the remaining groups were used for a second intramolecular cross-linking reaction (i.e., formally being type-B groups). This protocol allowed us to compare the structure of the resulting nanoparticles (A+B cross-linked 'heterofunctional' nanoparticles) with that of the 'homofunctional' nanoparticles, synthesized from exactly the same precursor polymer, when the intramolecular cross-linking process was carried out until completion exclusively via the Michael addition reaction (A cross-linked nanoparticles) or, alternatively, exclusively via intrachain metal complexation (B crosslinked nanoparticles). Experimental conditions for unimolecular soft nanoparticle formation through intrachain Michael addition reactions were previously reported in Ref. [2], whereas the synthesis of single-chain metallopolymer nanoparticles will be described in detail in a forthcoming article.

\section{B. Characterization techniques}

The precursors and the nanoparticles were characterized by means of SEC/MALLS techniques. SEC measurements were performed at $30{ }^{\circ} \mathrm{C}$ on an Agilent 1200 system equipped with PLgel $5 \mu \mathrm{m}$ Guard and PLgel $5 \mu \mathrm{m}$ MIXED-C columns, a differential refractive index (RI) detector (Optilab Rex, Wyatt) and a MALLS detector (Minidawn Treos, Wyatt). THF was used as eluent at a flow rate of $1 \mathrm{ml} / \mathrm{min}$, and data analysis was performed with the ASTRA Software from Wyatt.

The SAXS experiments were conducted on a Rigaku 3-pinhole PSAXS-L equipment operating at $45 \mathrm{kV}$ and $0.88 \mathrm{~mA}$. The MicroMax-002+ XRay Generator System is composed by a microfocus sealed tube source module and an integrated X-Ray generator unit which produces $\mathrm{CuK}_{\alpha}$ transition photons of wavelength $\lambda=1.54 \AA$. The flight path and the sample chamber in this equipment are under vacuum. The scattered X-Rays are detected on a 
two-dimensional multiwire X-Ray Detector (Gabriel design, 2D-200X). This gas-filled proportional type detector offers a $200 \mathrm{~mm}$ diameter active area with c.a. $200 \mathrm{mi}-$ cron resolution. The azimuthally averaged scattered intensities were obtained as a function of momentum transfer $q=4 \pi \lambda^{-1} \sin (\theta / 2)$, where $\theta$ is the scattering angle. Reciprocal space calibration was done using silver behenate as standard. Measurements were performed at room temperature with a sample to detector distance of $2 \mathrm{~m}$. The solutions (concentration: $8 \mathrm{mg} / \mathrm{mL}$, THF as solvent) were filling capillaries of $2 \mathrm{~mm}$ thickness fixed perpendicular to the beam. The data were background corrected by subtracting the result of a measurement on the capillary filled with THF and applying the proper transmission corrections.

\section{MODEL AND SIMULATION DETAILS}

Fig. 1 depicts the architecture of the precursor polymer. This consists of a linear backbone of $N_{\mathrm{b}}$ beads. One side group is attached to each of the $N_{\mathrm{b}}$ beads of the backbone. There are two kinds of side groups, which are randomly distributed along the backbone. A number $N_{l}$ of the side groups contain three beads. The corresponding free end beads are the cross-linkers. Accordingly, we define the fraction of linker side groups as $f=N_{\mathrm{l}} / N_{\mathrm{b}}$. The remaining $N_{\mathrm{b}}-N_{\mathrm{l}}$ side groups contain one single bead. Thus, the macromolecule has a total of $N=2\left(N_{\mathrm{b}}+N_{\mathrm{l}}\right)$ beads. This simple model aims to mimic qualitatively the structure of real precursor polymers. These usually contain bulky side groups (e.g., phenyl groups), some of them being functionalized with short branches ending in reactive groups. A bead in this qualitative model represents the center-of-mass of typically 2-4 carbons [44]. We investigated two models of the macromolecule: a 'homofunctional' polymer in which all linker beads are identical, and a 'heterofunctional' one in which there are two kinds of linker beads (denoted as A and B). We only considered the case of the same number, $N_{\mathrm{l}} / 2$, for both the A- and B-linkers, with their respective side groups being again randomly distributed along the backbone. The rest of the (non-reactive) beads in the macromolecule are denoted as $\mathrm{C}$.

The interaction between any two given beads, of the species $\alpha, \beta \in\{\mathrm{A}, \mathrm{B}, \mathrm{C}\}$, is given by a shifted LennardJones (LJ) potential

$$
V_{\alpha \beta}(r)=4 \epsilon_{\alpha \beta}\left[\left(\frac{\sigma_{\alpha \beta}}{r}\right)^{12}-\left(\frac{\sigma_{\alpha \beta}}{r}\right)^{6}+\frac{1}{4}\right],
$$

for $r<r_{\mathrm{c}}$, and $V_{\alpha \beta}(r)=0$ for $r \geq r_{\mathrm{c}}$. By using a cut-off distance $r_{\mathrm{c}}=2^{1 / 6} \sigma_{\alpha \beta}$, potential and forces are continuous at $r=r_{\mathrm{c}}$. Moreover they are purely repulsive and have no local minima. With this choice our implicitsolvent simulations mimic cross-linking in good solvent conditions. In addition to the Lennard-Jones potential, two connected monomers interact through a finitely ex-
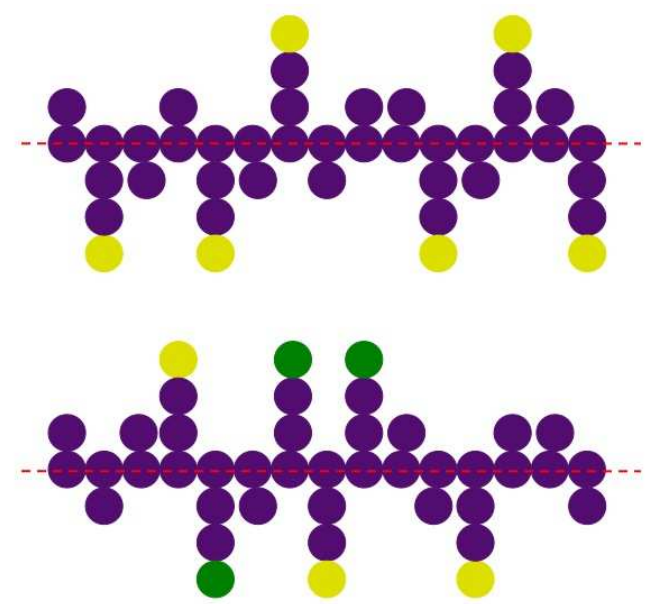

FIG. 1: Scheme of the simulated model for the homofunctionl (top) and heterofunctional precursor (bottom). The two types of linker beads (A and B) are yellow and green colored. Nonreactive $\mathrm{C}$-beads are indigo colored. The main backbone is indicated by the dashed line.

tensible nonlinear elastic potential (FENE) [44]:

$$
V_{\alpha \beta}^{\mathrm{FENE}}(r)=-\epsilon_{\alpha \beta} K_{\mathrm{F}} R_{0}^{2} \ln \left[1-\left(\frac{r}{R_{0} \sigma_{\alpha \beta}}\right)^{2}\right] \text {, }
$$

with $K_{\mathrm{F}}=15$ and $R_{0}=1.5$. The sum of the potentials (1) and (2) yields an effective potential between connected monomers with a deep minimum at $r=0.96 \sigma_{\alpha \beta}$, which guarantees chain uncrossability [44]. For simplicity we used the same energy and length scales of the interactions between the different species in Eqs. 1 and 2, namely $\epsilon_{\alpha \beta}=\epsilon=1$ and $\sigma_{\alpha \beta}=\sigma=1$ for $\alpha, \beta \in\{\mathrm{A}, \mathrm{B}, \mathrm{C}\}$.

We performed Langevin dynamics simulations. The equation of motion for each monomer $1 \leq i \leq N$, of mass $m_{i}$, is [45]:

$$
m_{i} \ddot{\mathbf{r}}_{i}=\mathbf{F}_{i}(t)-\gamma_{i} m_{i} \dot{\mathbf{r}}_{i}(t)+\mathcal{R}_{i}(t)
$$

The term $\mathbf{F}_{i}(t)$ is the Newtonian force resulting from the LJ and FENE interactions of $i$ with other monomers in its chain. The second and third terms in the right-hand side of Eq. 3 are the drag and random force respectively. The strength of the drag force is controlled by the friction term $\gamma_{i}$. The random forces are uncorrelated, following the relations $\left\langle\mathcal{R}_{i}^{\alpha}(t)\right\rangle=0$ and $\left\langle\mathcal{R}_{i}^{\alpha}(t) \mathcal{R}_{j}^{\beta}\left(t^{\prime}\right)\right\rangle=$ $2 \gamma_{i} m_{i} k_{\mathrm{B}} T \delta_{i j} \delta_{\alpha \beta} \delta\left(t-t^{\prime}\right)$, where greek indexes denote cartesian components. For simplicity we used indentical masses $m_{i}=m=1$ and frictions $\gamma_{i}=\gamma=0.05$ for all beads. The simulation time unit is $\tau=\left(m \sigma^{2} / \epsilon\right)^{1 / 2}$. The Langevin equations of motion were discretized with time step $\Delta t=0.01 \tau$ and integrated in the velocityVerlet scheme, following the impulse approach proposed in Refs. [46, 47]. Thus, coordinates $\mathbf{r}_{i}(t)$ and velocities 
$\dot{\mathbf{r}}_{i}(t)$ were propagated by iteration of Eqs. 4 to 7 :

$$
\begin{gathered}
\tilde{\mathbf{v}}_{i}=\dot{\mathbf{r}}_{i}(t)+\frac{\mathbf{F}_{i}(t)}{2 m_{i}} \Delta t \\
\mathbf{v}_{i}^{\prime}=e^{-\gamma_{i} \Delta t} \tilde{\mathbf{v}}_{i}+\sqrt{\frac{2 \gamma_{i} k_{\mathrm{B}} T}{m_{i}}} \mathbf{Z}_{i}^{(1)} \\
\mathbf{r}_{i}(t+\Delta t)=\mathbf{r}_{i}(t)+\frac{1-e^{-\gamma_{i} \Delta t}}{\gamma_{i} \Delta t} \tilde{\mathbf{v}}_{i}+\sqrt{\frac{2 k_{\mathrm{B}} T}{\gamma_{i} m_{i}}} \mathbf{Z}_{i}^{(2)} \\
\dot{\mathbf{r}}_{i}(t+\Delta t)=\mathbf{v}_{i}^{\prime}+\frac{\mathbf{F}_{i}(t+\Delta t)}{2 m_{i}} \Delta t
\end{gathered}
$$

The quantities $k_{\mathrm{B}}$ and $T$ are the Boltzmann constant and the imposed temperature, respectively. We used a value $k_{\mathrm{B}} T=1$ in all simulations. The vectors $\mathbf{Z}_{i}^{(k)}$ with $k=1,2$ are given by

$$
\left(\begin{array}{l}
\mathcal{Z}_{i}^{(1) \alpha} \\
\mathcal{Z}_{i}^{(2) \alpha}
\end{array}\right)=\left(\begin{array}{cc}
\sqrt{\tau_{2}} & 0 \\
\frac{\tau_{1}-\tau_{2}}{\sqrt{\tau_{2}}} & \sqrt{\Delta t-\frac{\tau_{1}^{2}}{\tau_{2}}}
\end{array}\right)\left(\begin{array}{l}
\Omega_{i}^{(1) \alpha} \\
\Omega_{i}^{(2) \alpha}
\end{array}\right)
$$

where $\alpha$ denotes cartesian components and $\tau_{k}=$ $\left(k \gamma_{i}\right)^{-1}\left[1-\exp \left(-k \gamma_{i} \Delta t\right)\right]$. The quantities $\Omega_{i}^{(k) \alpha}$ are, at each iteration, random numbers sampled from a Gaussian distribution of zero mean and variance equal to one.

The initial configuration of each precursor (unlinked) macromolecule was generated by sequential insertion of the beads and imposing a bond distance $r=\sigma$. The linear backbone of $N_{\mathrm{b}}$ beads was first generated and, for a fixed value of $N_{l}$, the two kinds of side groups were randomly distributed along the backbone. Random orientation was permitted for each new insertion, with the constraint $r>0.9 \sigma$ for the distance to all the previuosly inserted beads, preventing strong overlap. Equilibration runs were performed to unfold the initial conformations, and extended over several millions steps in order to sample equilibrium configurations of the precursors. Crosslinking was not activated (see below) during the equilibration runs.

In both the equilibration and cross-linking runs several realizations of the macromolecule were propagated simultaneously and independently (i.e., without intermolecular interactions). This aims to mimic intramolecular crosslinking of, highly-diluted, isolated macromolecules. The number $N_{\mathrm{m}}$ of independent macromolecules in each run was selected so that the total number of beads $N_{\mathrm{m}} N$ per run was of the order of $10^{4}$. This number was sufficient to achieve small temperature fluctuations and efficient thermalization at the selected $T$. For each value of the backbone length $N_{\mathrm{b}}$ and fraction of linker groups $f$, we performed $N_{\mathrm{R}}$ runs, producing configurations for
$N_{\mathrm{t}}=N_{\mathrm{R}} N_{\mathrm{m}}$ independent realizations of the intramolecular cross-linking process. Thus, statistical averages included average over both the $N_{\mathrm{t}}$ distinct macromolecules (ensemble-average), and the different configurations sampled by the propagation of the individual macromolecules (time-average). As will be shown in next sections, these two averages are not equivalent for the cross-linked polymers. Ensemble average was typically performed over $N_{\mathrm{t}}=200$ distinct macromolecules, though we used a large number $N_{\mathrm{t}}=1000$ for some selected cases. Timeaverage was performed over several hundreds of conformations for each individual macromolecule. These conformations were selected at equispaced times. The time ellapsed between selected consecutive conformations of a same macromolecule was long enough for uncorrelation. Indeed the average monomer displacement relative to the center-of-mass in that time interval was of the order of the average diameter of gyration.

The equilibrated precursors (see above) were used as the starting configurations for the cross-linking process. Likewise, we used initial sets of (canonically distributed) velocities obtained from the equilibration runs. Now we describe the implementation of the cross-linking process. A linker can only form one bond with other linker of its same species, i.e., in the case of the heterofunctional polymer A-B bonds are forbidden. We considered two types of cross-linking routes for the heterofunctional polymer, corresponding to two limit cases: simultaneous and sequential cross-linking. In the case of simultaneous crosslinking, both A- and B-linkers were simultaneously active (i.e., allowed to form bonds) from the beginning of the cross-linking run. In the case of sequential crosslinking, B-linkers were not active during the cross-linking of the A-linkers. Once the latter was completed, an equilibration run of a few million steps was performed and then cross-linking of the B-species was started. Obviously, for fixed $N_{\mathrm{b}}$ and $f$ the sequential cross-linking is computationally more demanding than the simultaneous one. Unless otherwise specificied, simulation observables will be reported for the simultaneous case. Sequential cross-linking was investigated for a few selected values of $\left(N_{\mathrm{b}}, f\right)$. As will be discussed in next sections, both cross-linking routes produced fully cross-linked nanoparticles with essentially the same structural properties.

Cross-linking in the simulations was irreversible. Thus, when a bond was formed between two given linkers, these remained bonded for the rest of the simulation (with the bonding interaction given by the FENE potential of Eq. 2), and were not allowed to form new bonds with other linkers. At a given time step, two linkers $i$ and $j$ of the same species can form a mutual bond only if the two following conditions are fulfilled: i) both of them are not bonded to any other linker; ii) they are at a mutual distance $r_{i j} \leq 1.3 \sigma_{i j}$ ('capture distance') [48]. For a given configuration, a list of all 'candidate' pairs $(i, j)$ fulfilling both conditions is made. Then, a pair $\left(i^{\prime}, j^{\prime}\right)$ is randomly selected from the list and a permanent bond is set between them. Since $i^{\prime}$ and $j^{\prime}$ cannot form new 
bonds, the other pairs eventually containing $i^{\prime}$ and $j^{\prime}$ are removed from the list of candidates. A new pair of the remaining candidates is randomly selected and the former procedure is repeated until the list becomes empty for the current configuration. Then the configuration is propagated according to the equations of motion and the former scheme is subsequently applied during the crosslinking run until no more bonds can be formed. One or zero linkers will remain unbonded at this point if $N_{\mathrm{l}}$ is odd or even, respectively.

Once cross-linking was completed, simulations were further extended over several millions steps to accumulate configurations for statistical averages. Verlet lists [49] were implemented in both equilibration and crosslinking runs, in order to reduce computational expense. We investigated cross-linking for a broad range of backbone lengths, $20 \leq N_{\mathrm{b}} \leq 1600$, and fractions of linker groups, $0.08<f<0.67$. Even for fixed values of $N_{\mathrm{b}}$ and $f$, the time $t_{\mathrm{cl}}$ needed for completing cross-linking showed a broad distribution for the different macromolecules, with a dispersion of about three time decades. This is a direct consequence of the highly stochastic character of the cross-linking process (see below). According to the number of beads per polymer, $2\left(N_{\mathrm{b}}+N_{\mathrm{l}}\right)$, the longest times extended from $t_{\mathrm{cl}}<10^{6}$ MD steps (for $N_{\mathrm{b}} \sim 20$ ) to $t_{\mathrm{cl}}>10^{8} \mathrm{MD}$ steps (for $N_{\mathrm{b}}=1600$ ). For the largest investigated systems there was a small fraction $(<10 \%)$ of macromolecules that did not complete cross-linking within the simulated time scales. These were excluded in the calculation of the radii of gyration and shape parameters presented in next sections, which strictly correspond to the fully cross-linked nanoparticles.

\section{SIMULATIONS: RESULTS AND DISCUSSION}

\section{A. Scaling behavior}

Fig. 2 shows simulation results for the averaged squared radius of gyration $\left\langle R_{\mathrm{g}}^{2}\right\rangle$, for all the investigated values of $N_{\mathrm{b}}$ and $f$. Here the brackets denote average over polymers and times (i.e., joint ensemble and time average). The results are shown for the investigated macromolecules in different states (unlinked precursor, homofunctional nanoparticle and heterofunctional nanoparticle). Each data set (fixed color and symbol code) corresponds to the $N_{\mathrm{b}}$-dependence of $\left\langle R_{\mathrm{g}}^{2}\right\rangle$ for a same state and a same $f$. It must be noted that the precursor states for the homofunctional and heterofunctional polymers are equivalent, since the interaction potentials between all species are identical (see Section III). Differences between homofunctional and heterofunctional polymers with the same $N_{\mathrm{b}}$ and $f$ arise only in the cross-linked states. This is a consequence of the fact that, unlike in the homofunctional polymers, in the heterofunctional ones half of the linkers cannot form bonds with the other half.

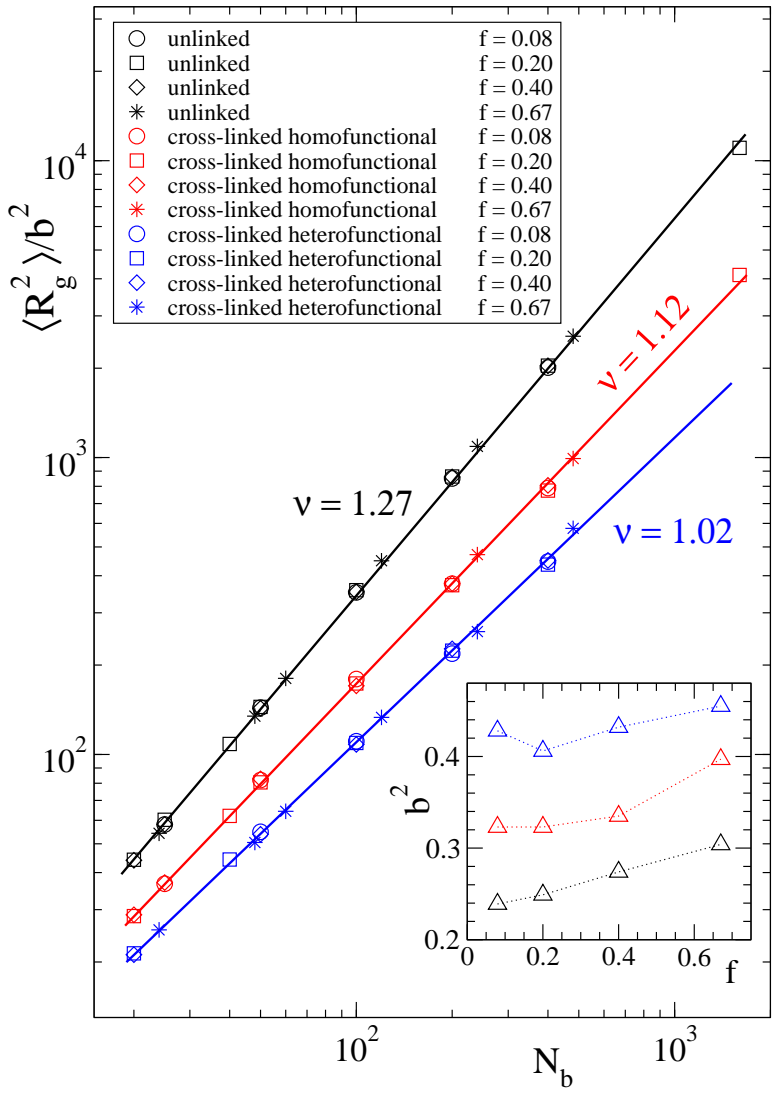

FIG. 2: Symbols in main panel are the average radius of gyration vs. backbone length $N_{\mathrm{b}}$ for all the investigated linker fractions $f$. Data are normalized by the squared length $b^{2}$ of the effective segment, obtained from fits (solid lines) to power laws $\left\langle R_{\mathrm{g}}^{2}\right\rangle=b^{2} N_{\mathrm{b}}^{\nu}$ (see text). The $\nu$-exponents are indicated. The symbols in the inset are the obtained values of $b^{2}$ vs. the fraction of cross-linkers $f$. Same symbol codes in main panel correspond to same $f$-values (see legend). Color codes in both main panel and inset correspond to the cases of unlinked precursors (black), cross-linked homofunctional polymers (red), and cross-linked heterofunctional polymers (blue).

The different data sets in Fig. 2 are consistent with power-law scaling $\left\langle R_{\mathrm{g}}^{2}\right\rangle=b^{2} N_{\mathrm{b}}^{\nu}$, with $b$ the effective segment length and $\nu$ the effective exponent. Free fits (not shown) give essentially the same exponent for a same state, irrespectively of the fraction of linker groups. Therefore, for each state we have fixed the value of $\nu$ to the average over the different fractions $f$ for such a state, and repeated the fits by leaving $b^{2}$ as the only free parameter. The different data sets in Fig. 2 are normalized by the so-obtained values of $b^{2}$. By using this representation, data for all the linker fractions of a same state show an excellent agreement with a common power law. The representation also reflects a very different scaling behavior for the different states. The scaling exponent for the unlinked precursor is $\nu=1.27$. This is bigger than the Flory exponent $\left(\nu_{\mathrm{F}}=1.18\right)[50,51]$ for self-avoiding random walks, which describe the conformations of linear chains in good solvent. In principle, the unlinked precur- 

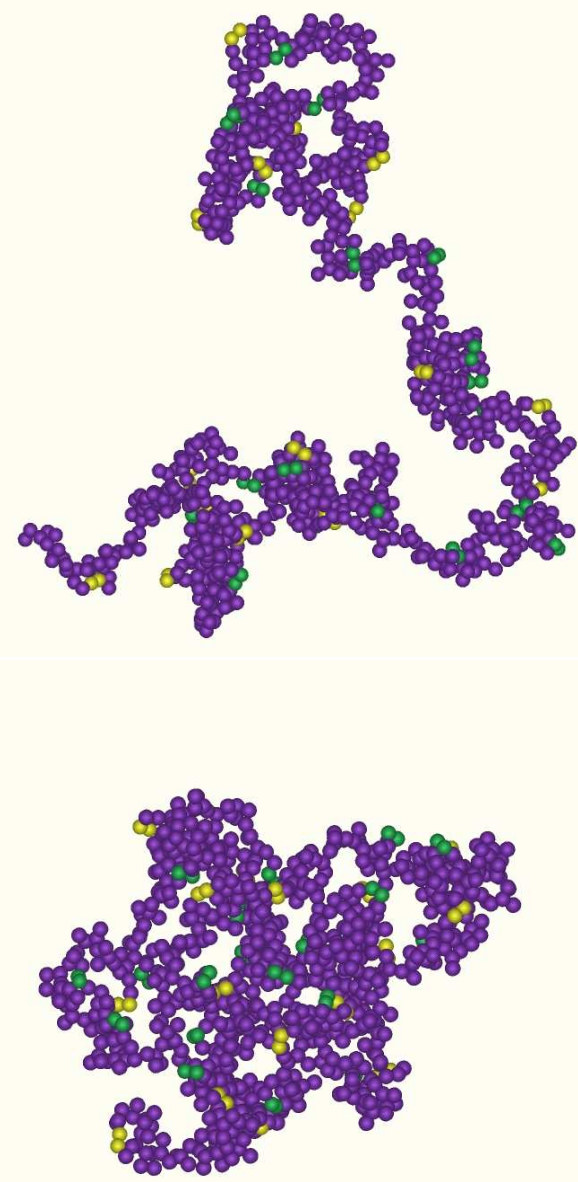

FIG. 3: Typical snapshots of two distinct cross-linked comopolymers of $N_{\mathrm{b}}=400$ and $f=0.20$, with open (top) and more compact (bottom) topologies. The two types of linkers are depicted in yellow and green color.

sors should converge to this behavior for sufficiently large values of $N_{\mathrm{b}}$. The observed scaling with $\nu=1.27$ might be an intermediate regime related to the effective stiffness of the backbone induced by the side groups, leading to an increase of the size with the molecular weight faster than what is expected for flexible linear chains.

The nanoparticles exhibit significantly lower exponents, $\nu=1.12$ and 1.02 for the homofunctional and heterofunctional case respectively. The exponent for the heterofunctional nanoparticles is essentially identical to that of random walks $\left(\nu_{\mathrm{RW}} \equiv 1\right)$, which describe the (Gaussian) statistics of linear chains in polymer melts or in $\theta$-solvents [50, 51]. The observed crossover in the scaling behavior by fully cross-linking the precursor slightly resembles a transition to a $\theta$-point. Indeed we will show that, as for the case of linear chains in $\theta$-solvent, the nanoparticles show a local globulation of the monomers but their conformations remain open at the large scale.
Local globulation is anticipated by the increase of the effective segment length $b$ observed by passing from the unlinked to the fully cross-linked state (inset of Fig. 2). Open conformations are suggested by the scaling exponents. Indeed if the dominant conformations of the crosslinked polymers were compact, they should lead to lower exponents (e.g., $\nu=2 / 3$ for spherical objects) than those observed in Fig. 2. This feature is confirmed by visual inspection of the nanoparticles. Fig. 3 shows representative snapshots for the heterofunctional nanoparticles with $N_{\mathrm{b}}=400$ and $f=0.20$. The irreversible process of full cross-linking can produce both open structures (top panel) and more compact ones (bottom panel), though the second ones are still sparse. Analogous observations are found for all the investigated backbone lengths and linker fractions.

The underlying mechanism leading to sparse nanoparticles can be understood by analyzing the contour distances between bonded linkers. We define the contour distance $s$ between two bonded linkers as $1+n$, with $n$ the number of backbone monomers between the two backbone monomers to which the corresponding linker side groups are attached. Fig. 4 shows the time evolution of the histogram $P(s)$ during the cross-linking process. Data are shown for a representative case, $N=200$ and $f=0.40$, of the homofunctional polymer. We observe that $P(s)$ is a monotonically decreasing function of $s$. Thus, bonds between linkers at small $s$ are much more likely to be formed that at large countour distances. Moreover, bonds with large $s$ are mostly formed at the late stage of the cross-linking process. These features can be understood as follows. Bonds are formed between pairs of linkers that are obviously close in the real space. Because of the self-avoiding character of the unlinked precursors (good solvent conditions), such linkers will be mostly at a small contour distance from each other. Folded conformations of the unlinked precursor, in which two linkers are far from each other in contour distance, but close in real distance, do occur but are much less frequent. For that reason the early stage of the crosslinking process is dominated by bonding between pairs at small $s$. By looking in detail at the different data sets of Fig. 4, it is found that for small contour distance, $s \lesssim 7, P(s)$ grows up until $t \sim 400$ and no significant increase occurs beyond that time scale. By increasing the time beyond $t \sim 400$, the distribution $P(s)$ grows up at intermediate scales. For the selected case of Fig. 4, no significant increase of $P(s)$ is observed in the range $s \lesssim 22$ for $t \gtrsim 4000$. Thus, the interval $400 \lesssim t \lesssim 4000$ corresponds to bonding between linkers at progressively longer contour distances, from $s \sim 7$ to $s \sim 22$. Finally, the late stage of the cross-linking process reflects the formation of bonds between linkers separated by large contour distances. This is shown in Fig. 4 by the ultimate growth of $P(s)$ for $s \gtrsim 22$, from $t \sim 4000$ to the end of the simulation at $t \sim 4.6 \times 10^{5}$. This ultimate regime essentially corresponds to bonding between the last pairs of unbonded linkers. Fig. 4 shows that these 


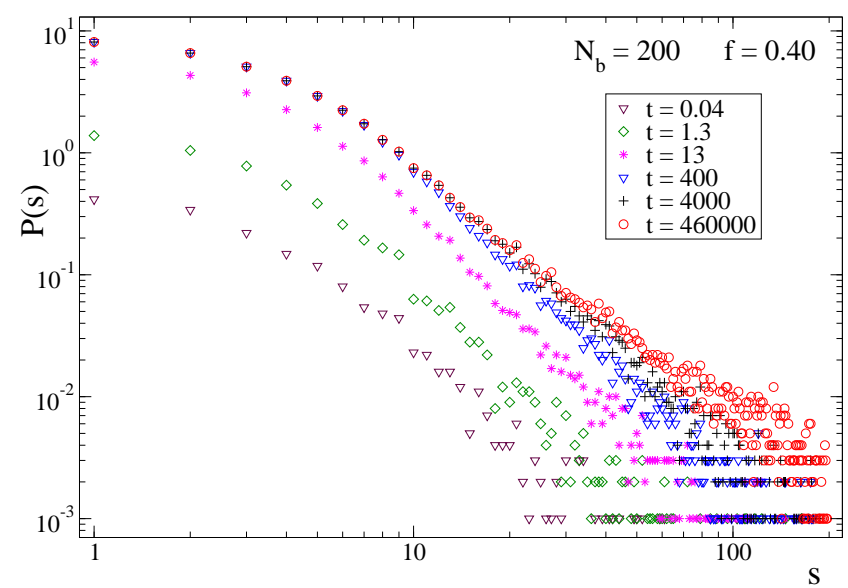

FIG. 4: Time evolution of the histogram of contour distances $s$ between bonded linkers, for the homofunctional polymer of $N_{\mathrm{b}}=200$ and $f=0.40$. Different data sets correspond to different selected times (see legend).

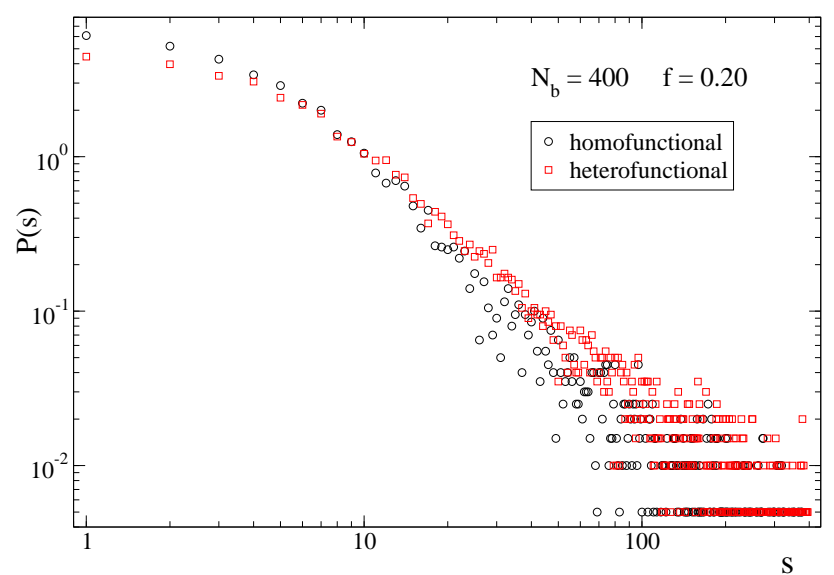

FIG. 5: Histogram of contour distances $s$ between bonded linkers for fully cross-linked nanoparticles with identical values of $N_{\mathrm{b}}=400$ and $f=0.20$. Circles and squares correspond respectively to the homofunctional and heterofunctional case.

linkers are on average at large contour distances. This requires large scale reorientation of the polymer to put the remaining unbonded linkers into contact. Because of the highly stochastic character of such large reorientations, the time for completing cross-linking can vary by orders of magnitude for polymers of identical $N_{\mathrm{b}}$ and $f$.

Fig. 5 compares results, for fixed $N_{\mathrm{b}}=400$ and $f=0.20$, of the distribution $P(s)$ for the homofunctional and heterofunctional nanoparticles. Bonding at short countour distances is also clearly dominant in the heterofunctional case. However, bonding at long $s$ is more likely than in the homofunctional counterparts. This can be understood as follows. If a homofunctional polymer of backbone length $N_{\mathrm{b}}$ has $N_{\mathrm{l}}$ linkers, its heterofunctional counterpart of identical $N_{\mathrm{b}}$ has $N_{\mathrm{l}} / 2$ A-linkers and other $N_{\mathrm{l}} / 2$ B-linkers, both types of linkers being randomly distributed along the precursor contour. Since in the hetero- functional polymer A-B bonds are forbidden, the average contour distance between linkers that can form mutual bonds (A-A or B-B pairs) will be larger than in the homofunctional counterpart. This will lead, in the distribution $P(s)$ of the heterofunctional polymer, to a lower amplitude at small $s$ and a less pronounced decay at long $s$, respect to the $P(s)$ of the homofunctional polymer.

The results displayed in Figs. 4 and 5 rationalize the trends observed in the scaling behavior of the radius of gyration (Fig. 2). Bonding at short contour distances leads to compactation of the chain at local scales by creating small dense loops. However, this is not an efficient mechanism for compactation at large scales. On the contrary, looping over long countour distances does lead to a significant reduction of the macromolecular size - the most simple case is linking the two end monomers of a linear chain to form a ring. However, because of the self-avoiding character of the precursor chains in good solvent conditions, bonding between linkers at long $s$ is unfrequent. Hence the average number of long loops in the fully cross-linked nanoparticle is very small and at the end of the synthesis process does not lead to strong compactation at large scales. Still, size reduction respect to the precursor is more pronounced for the heterofunctional than for the homofunctional nanoparticle. This feature is rationalized by the higher probability in the heterofunctional polymer for forming long-s bonds. As a consequence of the local, but not global, compactation of both the homofunctional and heterofunctional nanoparticles, the scaling exponents for $\left\langle R_{\mathrm{g}}^{2}\right\rangle$ are much closer to that of chains in $\theta$-solvent $(\nu=1)$ than to that of globular polymers $(\nu=2 / 3)$.

As demonstrated by visual inspection (Fig. 3), irreversible cross-linking of polymers with same $N_{\mathrm{b}}$ and $f$ can lead to nanoparticles of rather different topologies. This suggests that ensemble and time average (as defined above) are not equivalent. We denote by $R_{\mathrm{g}}$ the instantaneous values of the radii of gyration adopted by the polymers at different time steps, and by $\bar{R}_{\mathrm{g}}$ the time averages of such values for the individual polymers. Though, because of the random distribution of the linker branches along the backbone, the architectures of the different precursors are not strictly equivalent for fixed $N_{\mathrm{b}}$ and $f$, this has a negligible effect in the time average. Thus, all precursors with the same $N_{\mathrm{b}}$ and $f$ have the same $\bar{R}_{\mathrm{g}}$ within statistics. On the contrary, the rather different topologies adopted by nanoparticles with the same $N_{\mathrm{b}}$ and $f$ lead to a distribution of $\bar{R}_{\mathrm{g}}$. Fig. 6 shows results for the distributions of both the instantaneous and timeaveraged radii of gyration, $P\left(R_{\mathrm{g}}\right)$ and $P\left(\bar{R}_{\mathrm{g}}\right)$ respectively. Results correspond to the unlinked precursor, homofunctional nanoparticle and heterofunctional nanoparticle, all them with identical backbone length $N_{\mathrm{b}}=200$ and linker fraction $f=0.20$. As mentioned before, the different precursors show the same value of $\bar{R}_{\mathrm{g}}$, in spite of the broad distribution of instantaneous $R_{\mathrm{g}}$ that is explored by intramolecular fluctuations. Instead, the nanoparticles are intrinsically polydisperse in size. The intramolec- 


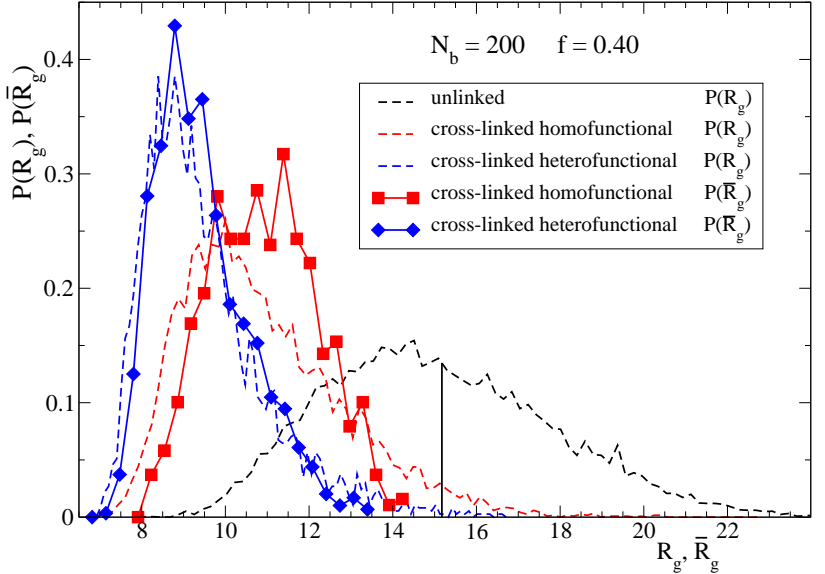

FIG. 6: Dashed lines: for systems with $N_{\mathrm{b}}=200$ and $f=0.40$, distributions of instantaneous values of the radius of gyration $R_{\mathrm{g}}$ in the precursor and cross-linked homofunctional and heterofunctional polymer. Symbols joined by solid lines are the distributions of the corresponding values of the time-averaged radius of gyration $\bar{R}_{\mathrm{g}}$ for the homofunctional (squares) and heterofunctional polymer (diamonds). The solid (black) vertical line indicates the single value of $\bar{R}_{\text {g }}$ for the unlinked precursor.

ular fluctuations lead to different time-averaged radii of gyrations for the different polymers. Consistently with the trends observed for the joint (time and ensemble) averages $\left\langle R_{\mathrm{g}}^{2}\right\rangle$ in Fig. 2, there is a shift of the distributions to lower sizes by passing from the unlinked state to the cross-linked one. Likewise, the distributions for the heterofunctional nanoparticles are shifted to lower sizes respect to those of the homofunctional counterparts. The first ones are also narrower, but this seems to be just an effect of size reduction. Indeed heterofunctional and homofunctional nanoparticles seem to have the same intrinsic polydispersity. Thus, if we characterize the polydispersity by the ratio $\sigma_{\mathrm{R}}=\left[\left\langle\bar{R}_{\mathrm{g}}^{2}\right\rangle-\left\langle\bar{R}_{\mathrm{g}}\right\rangle^{2}\right]^{1 / 2} /\left\langle\bar{R}_{\mathrm{g}}\right\rangle$, we find within statistics the same 'half-width' $\sigma_{\mathrm{R}} \approx 12 \%$ for the heterofunctional and homofunctional case.

\section{B. Shape parameters}

More detail about the average macromolecular conformations can be obtained by quantifying shape parameteres as the asphericity and prolateness. These can be obtained from the radius of gyration tensor $[52,53]$,

$$
T_{\alpha \beta}=\frac{1}{N^{2}} \sum_{i=1}^{N}\left(r_{i \alpha}-r_{\alpha}^{\mathrm{cm}}\right)\left(r_{i \beta}-r_{\beta}^{\mathrm{cm}}\right) \text {, }
$$

where $\alpha, \beta$ denote cartesian components of the position vectors, $\mathbf{r}_{i}$ and $\mathbf{r}^{\mathrm{cm}}$ for the $i$ th-monomer and center-ofmass of the polymer, respectively. In the following we sort the three eigenvalues of the matrix of Eq. 9 as $\lambda_{1} \leq$ $\lambda_{2} \leq \lambda_{3}$. It can be seen that $R_{\mathrm{g}}^{2}=\lambda_{1}+\lambda_{2}+\lambda_{3}$. The

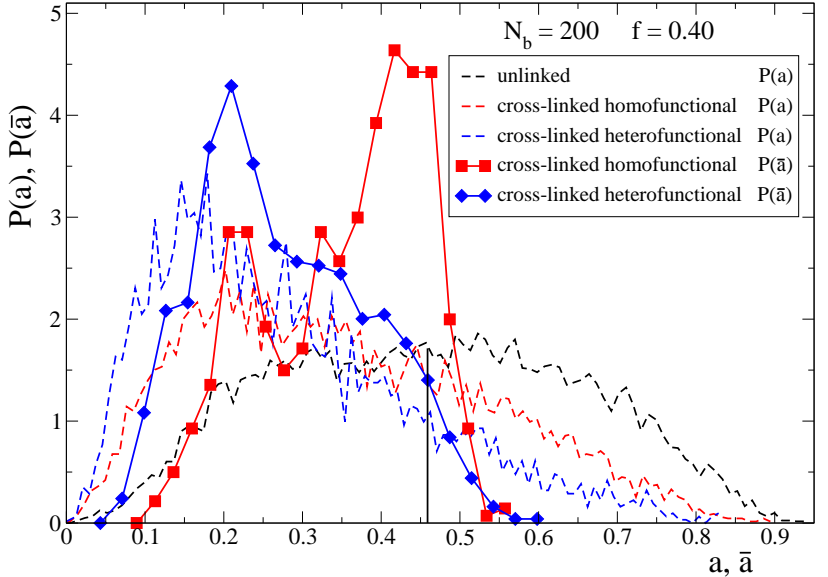

FIG. 7: As Fig. 6 for the asphericity parameters $a$ and $\bar{a}$.

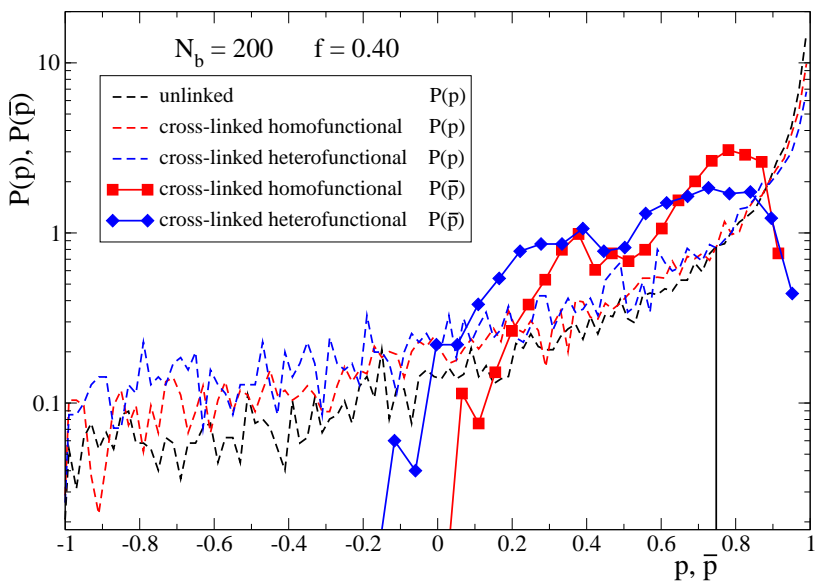

FIG. 8: As Fig. 7 for the prolateness parameters $p$ and $\bar{p}$.

asphericity parameter, $0 \leq a \leq 1$, is defined as:

$$
a=\left\langle\frac{\left(\lambda_{2}-\lambda_{1}\right)^{2}+\left(\lambda_{3}-\lambda_{1}\right)^{2}+\left(\lambda_{3}-\lambda_{2}\right)^{2}}{2\left(\lambda_{1}+\lambda_{2}+\lambda_{3}\right)^{2}}\right\rangle .
$$

The asphericity of a perfectly spherical object is $a=0$. Objects with approximate spherical shape $\left(\lambda_{1} \approx \lambda_{2} \approx\right.$ $\lambda_{3}$ ) have asphericities $a \gtrsim 0$. The prolateness parameter, $-1 \leq p \leq 1$, is defined as:

$$
p=\left\langle\frac{\left(3 \lambda_{1}-R_{\mathrm{g}}^{2}\right)\left(3 \lambda_{2}-R_{\mathrm{g}}^{2}\right)\left(3 \lambda_{3}-R_{\mathrm{g}}^{2}\right)}{2\left(\lambda_{1}^{2}+\lambda_{2}^{2}+\lambda_{3}^{2}-\lambda_{1} \lambda_{2}-\lambda_{1} \lambda_{3}-\lambda_{2} \lambda_{3}\right)^{3 / 2}}\right\rangle
$$

For perfectly oblate objects $\left(\lambda_{1}<\lambda_{2}=\lambda_{3}\right)$ the prolateness is $p=-1$. For perfectly prolate objects $\left(\lambda_{1}=\lambda_{2}<\right.$ $\left.\lambda_{3}\right) p=1$.

In analogy with the analysis presented in Fig. 6 for the radii of gyration, and for the same choice of parameters, we show in Figs. 7 and 8 the corresponding distributions of the instantaneous $(a, p)$ and time-averaged $(\bar{a}, \bar{p})$ asphericities and prolateness. The distributions of the asphericities show the same qualitative trends as those of the radii of gyration. Nanoparticles are, on average, 

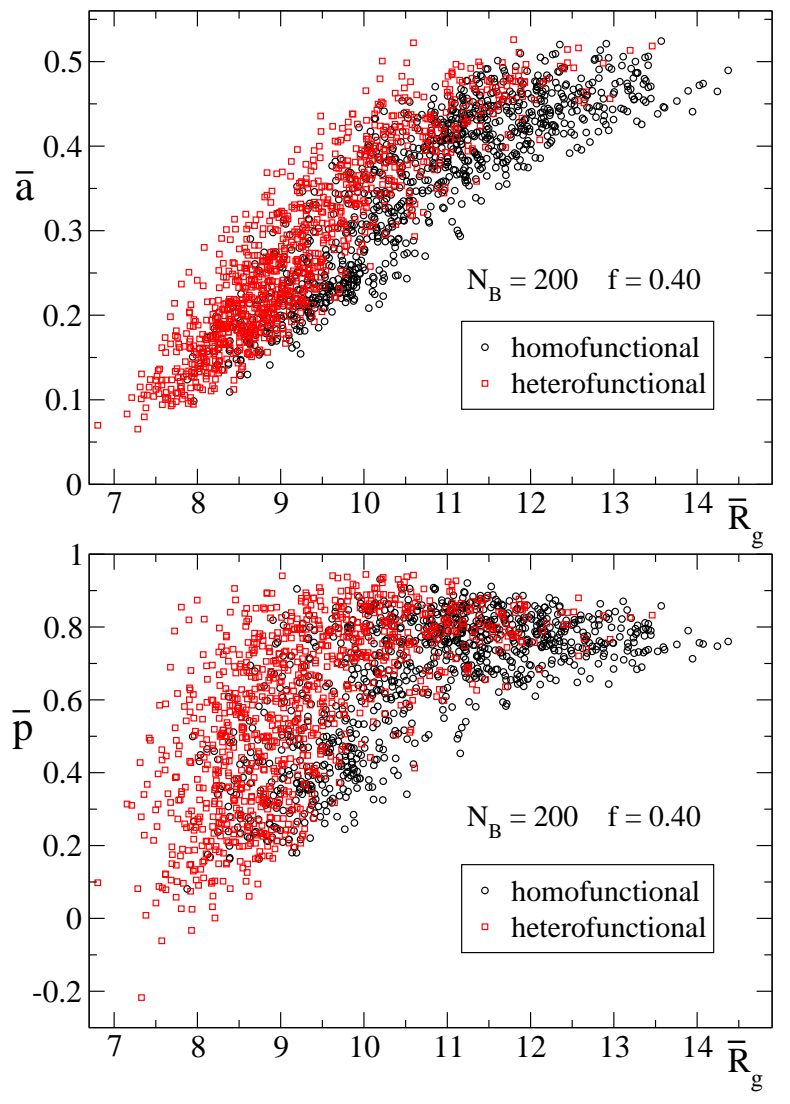

FIG. 9: Correlation of the time-averaged shape parameters $\bar{a}$ (top) and $\bar{p}$ (bottom) with the time-averaged radii of gyration $\bar{R}_{\mathrm{g}}$, for homofunctional (circles) and heterofunctional nanoparticles (squares) of $N_{\mathrm{b}}=200$ and $f=0.40$.

closer to spherical objects (lower asphericity) than the unlinked precursors, and this effect is more pronounced for the heterofunctional nanoparticles than for their homofunctional counterparts. Still, the contribution of quasispherical objects $(a \rightarrow 0)$ to the total distribution is rather small, as anticipated by visual inspection of the polymer conformations (Fig. 3). Though some big loops can be formed, leading to strong reduction of the polymer size respect to the unlinked state, the nanoparticles still exhibit extended portions which allow to explore highly non-spherical conformations. The distributions of the time-averaged asphericities exhibit, within statistics, an apparent bimodal character, which is more pronounced for the cross-linked homofunctional polymers. This bimodality reflects the presence of both open topologies (without big loops) and moderately compact ones, as the two representative cases illustrated in Fig. 3. The strong reduction of the large- $\bar{a}$ mode in the $P(\bar{a})$ of the heterofunctional nanoparticles suggests that open topologies are less frequent than in the homofunctional counterparts.

Concerning the distribution of prolateness (Fig. 8), this is largely dominated by prolate objects $(p \rightarrow 1)$ in all cases. Cross-linking just leads to a slight increase
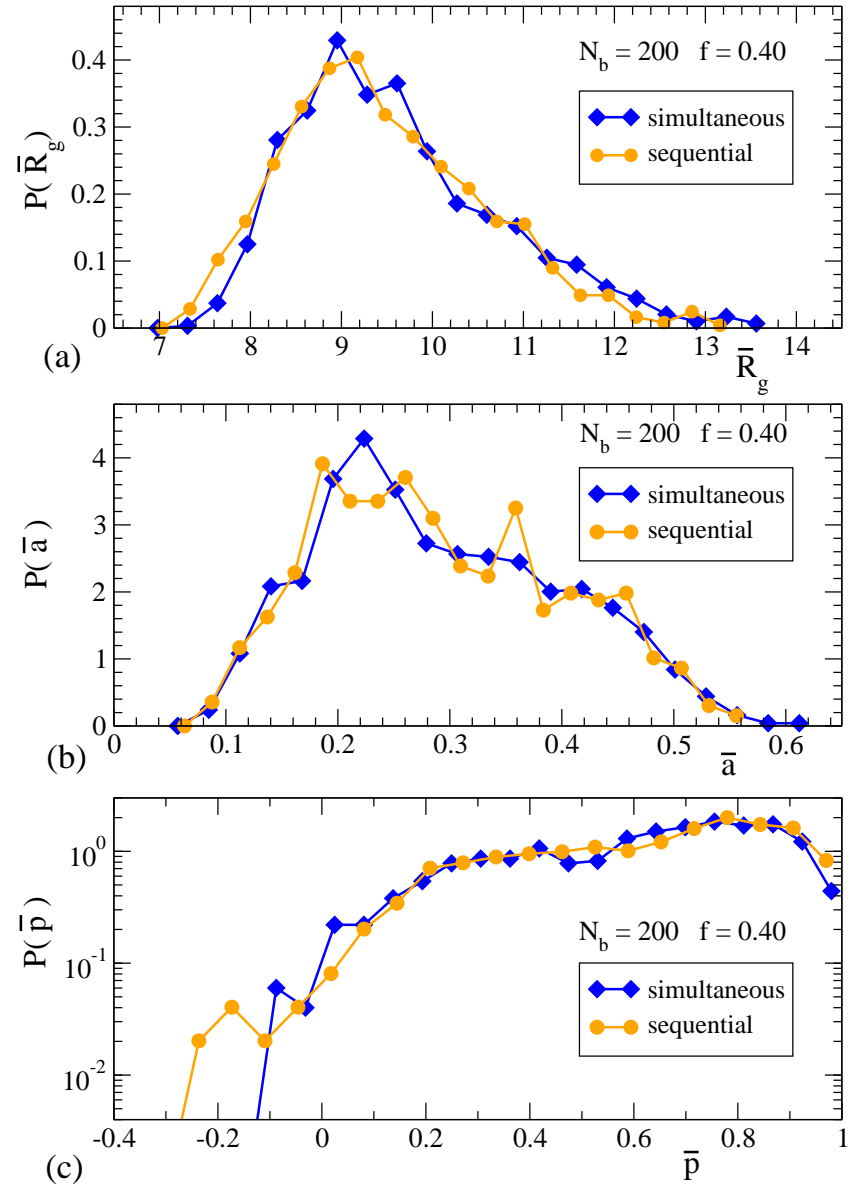

FIG. 10: Distributions of time-averaged values for size and shape parameters. (a): $P\left(\bar{R}_{\mathrm{g}}\right)$; (b): $P(\bar{a}) ;(\mathrm{c}): P(\bar{p})$. Data in all panels correspond to heterofunctional nanoparticles with identical values of $N_{\mathrm{b}}=200$ and $f=0.40$, but different crosslinking routes. Diamonds and circles correspond respectively to the simultaneous and sequential route.

in the probability of finding instantaneous oblate shapes $(p \rightarrow-1)$ by intramolecular fluctuations - note the logarithmic scale in Fig. 8. However, the distributions of the time-averaged prolateness, $P(\bar{p})$ reveal that intrinsicaly oblate topologies are extremely unfrequent.

Finally, Fig. 9 shows, for the former values $N_{\mathrm{b}}=200$ and $f=0.40$, the correlation between the time-averaged shape parameters obtained for the individual nanoparticles and their respective time-averaged radii of gyrations. The correlations seem to be more pronounced for the asphericity than for the prolateness. The representations of Fig. 9 show that, in general, the topologies of the crosslinked polymers become more spherical and less prolate for intrinsically smaller sizes (i.e., for lower $\bar{R}_{\mathrm{g}}$ ). 


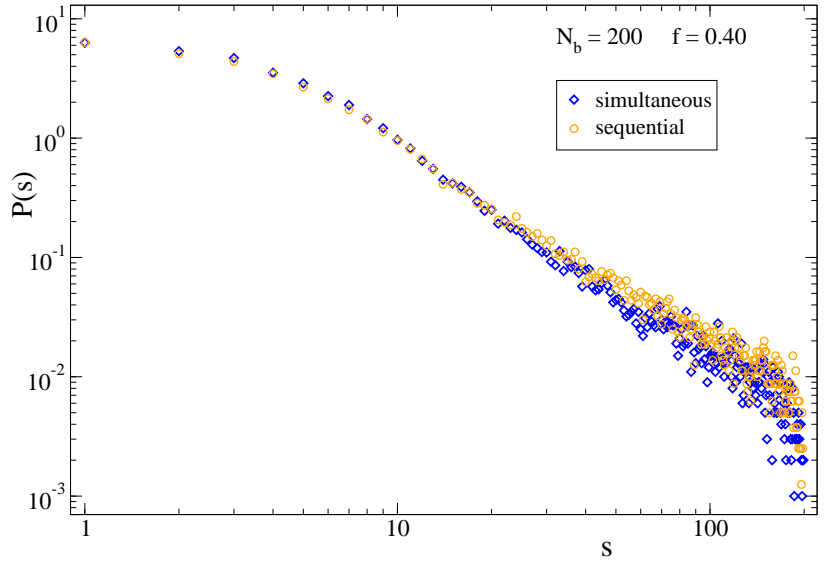

FIG. 11: Histogram of contour distances $s$ for heterofunctional nanoparticles with identical values of $N_{\mathrm{b}}=200$ and $f=0.40$, but different cross-linking routes. Diamonds and circles correspond respectively to the simultaneous and sequential route.

\section{Conformations of heterofunctional nanoparticles: simultaneous vs. sequential cross-linking}

The results presented for the heterofunctional polymers in previous subsections correspond to the case of simultaneous cross-linking of the two species A and B. In this subsection we discuss the effect of the specific cross-linking route (simultaneous or sequential) on the size and shape of the obtained heterofunctional nanoparticles. Fig. 10 compares the corresponding distributions of time-averaged radii of gyration, asphericities and prolateness for the same selected values $N_{\mathrm{b}}=200$ and $f=0.20$ of Figs. 6 to 8 . Likewise, Fig. 11 compares the corresponding histograms $P(s)$ of countour distances between bonded linkers in the heterofunctional nanoparticles. Differences between the distributions and histograms obtained by the simultaneous and sequential routes are negligible within statistics. As mentioned above, simultaneous cross-linking is less demanding computationally, whereas chemical synthesis has been performed by sequential cross-linking. The fact that both routes produce the same structural properties for the obtained cross-linked polymers supports the simulation of the simultaneous route as a robust and consistent methodology for guidance of experimental synthesis.

\section{Euler characteristic}

Results in the previous subsections characterize global properties of the macromolecular conformations. In order to further define the structure of the intramolecularly cross-linked polymers, taking into account multibody correlations among the constituent monomers, we use the Euler characteristic, $\chi$, as a topological fingerprint. This morphometric approach has been proven to be robust and suitable in bringing forward quantitative details of the spatial organization of the molecules [5456]. The Euler characteristic $\chi$ is one of the four scalar Minkowski functionals [57-59] that characterize a given surface embedded in three dimensions, the others being the enclosed volume, total surface area and the integral mean curvature. The coefficient itself is proportional to the integral Gaussian curvature and its value is not affected by any continuous, topology-preserving deformations of the surface. In three dimensions it takes the form:

$$
\chi=\mathcal{N}_{D}+\mathcal{N}_{C}-\mathcal{N}_{T} .
$$

$\mathcal{N}_{D}$ represents the number of disconnected aggregates, $\mathcal{N}_{C}$ is the number of enclosed cavities and $\mathcal{N}_{T}$ is the number of perforations, i.e., tunnels that percolate through the system. Values for some archetypical topologies are: i) $n$ for $n$ disconnected spherical polyhedra, ii) $1-n$ for a $n$-fold torus, and iii) $1+n$ for a sphere with $n$ inner holes.

For computing the Euler characteristic we proceeded as follows. As a first step we constructed a cubic box containing the macromolecule, and discretized the box into a cubic lattice, of spacing $d$. Each lattice site is surrounded by a Wigner-Seitz cell of the cubic lattice, i.e., by an elementary cube having the size of the lattice constant. This construction is space-filling. We then introduced a family of surfaces parameterized by an appropriate length scale $R$ that can be constructed from a given configuration of the monomers. To this end, we considered the surfaces $\mathcal{S}(R)$ formed by spheres with a radius $R$ located at the centers of every monomer. We denote each cell inside $\mathcal{S}(R)$ as 'full' and all others sites as 'empty'. Once $\mathcal{S}(R)$ was constructed, the Euler characteristic was computed as explained in Ref. [57].

In the limit of small $R$ we have a collection of disjoint spheres. By increasing $R$, some of the spheres will connect, merge, and later form rings and cavities. Eventually, the collection of spheres will fully occupy the space containing the macromolecule, and the surface will vanish. We stress that the so-obtained Euler characteristic corresponds to a discretized configuration of the surface $\mathcal{S}(R)$. Therefore, the lattice spacing $d$ must be sufficiently small to avoid artifacts arising from the discretization. For each value of $R$ we have controlled the sensitivity of the results to the selected values of $d$, finding an upper value $d_{0}(R)$ below which the obtained $\chi(R)$ is $d$-independent within statistics. In other words, for $d \leq d_{0}(R)$ the Euler characteristic is undistinguishable from that of the real surface $(d \rightarrow 0)$. Results presented in Fig. 12 have been calculated by using $d=d_{0}(R)$, for computational efficiency. For radii $R>1$, we have used $d_{0}(R)=0.2$. For $R<1$ smaller spacing is necessary, in particular around the minimum of $\chi(R)$, where we have used $d_{0}(R)=0.05$.

In Fig. 12 we plot $\chi(R)$ for selected values of $N_{\mathrm{b}}$ and $f$. Data in panels (a) and (b) correspond exclusively to the case of homofunctional polymers. Panel (a) shows re- 

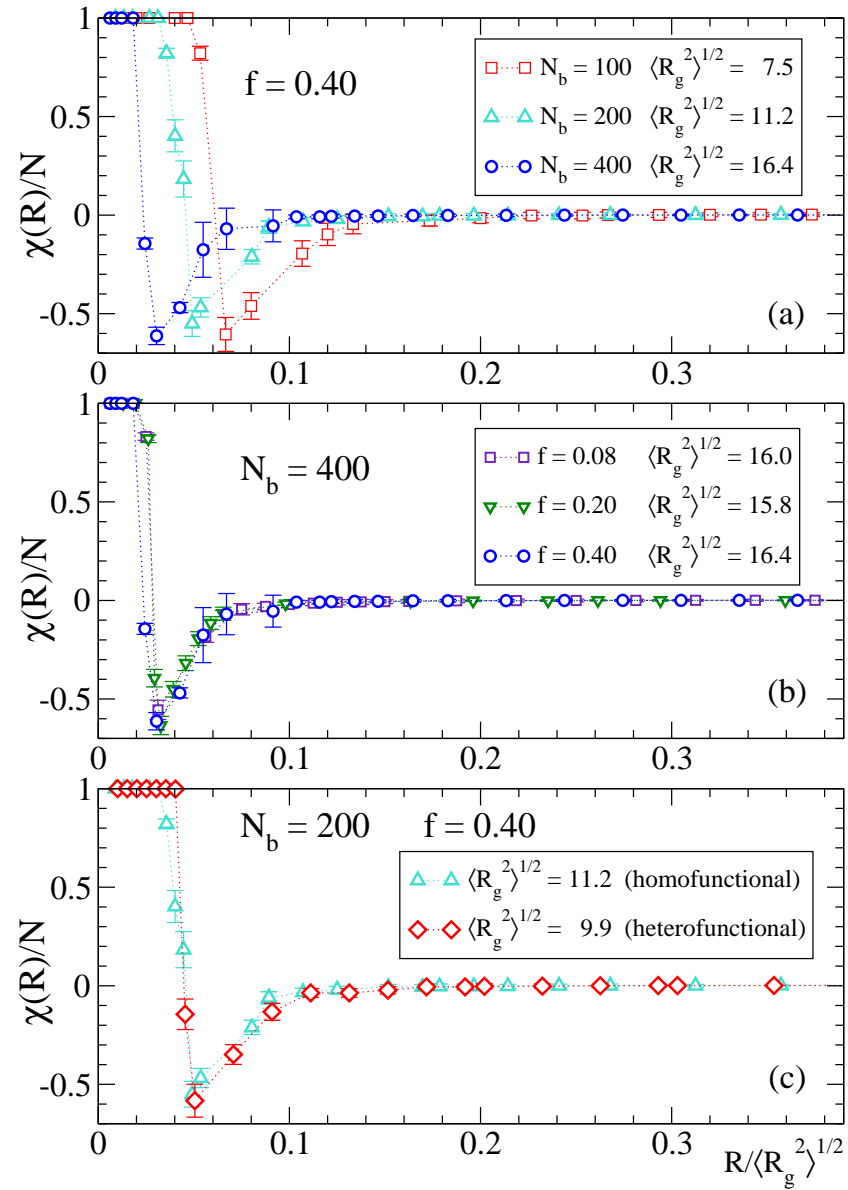

FIG. 12: Euler characteristic, $\chi(R)$, of the nanoparticles. Data in panels (a) and (b) correspond exclusively to the case of homofunctional polymers. (a): Results for fixed fraction of linkers $f=0.40$, and several values of the backbone length $N_{\mathrm{b}}$. (b): Results for fixed $N_{\mathrm{b}}=400$ and several values of $f$. (c): Comparison between homofunctional and heterofunctional polymers for fixed $N_{\mathrm{b}}=200$ and $f=0.40$. In all panels $\chi(R)$ is normalized by the number $N$ of monomers per polymer, and the distance is normalized by $\left\langle R_{\mathrm{g}}^{2}\right\rangle^{1 / 2}$ (see legends).

sults for several values of the backbone length $N_{\mathrm{b}}$ at fixed fraction of linkers $f=0.40$. Panel (b) shows results for several values of $f$ at fixed $N_{\mathrm{b}}=400$. Finally, in panel (c) we compare, for fixed $N_{\mathrm{b}}=200$ and $f=0.40$, the Euler characteristic of the homopofunctional nanoparticles and their heterofunctional counterparts. Results in all panels are ensemble and time-averaged. For comparison between macromolecules of different size and molecular weight, distances $R$ have been normalized by $\left\langle R_{\mathrm{g}}^{2}\right\rangle^{1 / 2}$ (see values in the legends of Fig. 12), and Euler characteristics $\chi(R)$ have been normalized by the total number, $N$, of monomers per polymer. With this normalization, we find by construction $\chi(R=0)=1$.

While we increase the radius $R$ of the spheres, the Euler characteristic changes if the topology of the surface $\mathcal{S}(R)$ changes as well. According to the definition of Eq. 12, there are two different mechanisms that decrease the value of $\chi(R)$ by increasing the radius $R$. The first mechanism is the external touching of two spheres, in which case either the number of disjoint surfaces is diminished (leading to a decrease of $\mathcal{N}_{D}$ ) or a chain of joined spheres forms a new loop (leading to an increase of $\mathcal{N}_{T}$ ). Both features result in negative contributions to $\chi$ in Eq. 12. The second mechanism is the disappearence of a cavity enclosed by at least four spheres, which decreases $\mathcal{N}_{C}$ in Eq. 12. On the other side, one event typically increases the value of $\chi(R)$ by growing $R$ : when three spheres forming a loop become so large than the hole of the corresponding torus or handle vanishes. This decreases the number of percolating tunnels $\mathcal{N}_{T}$, resulting in a positive contribution to $\chi$ in Eq. 12 .

Now we analyze the $R$-dependence of $\chi$ on the basis of these mechanisms. All data sets in Fig. 12 exhibit the same trends. Differences in panel (a) are just given by the macromolecular sizes (note the scaling of $R$ by $\left\langle R_{\mathrm{g}}^{2}\right\rangle^{1 / 2}$ ), and not by topological features. By starting from $R=0$, when the radius of the spheres approach to half of the typical bond length $(R \approx 0.5)$, the spheres merge into a single aggregate $\left(\mathcal{N}_{D}=1\right)$. This happens in a very narrow range of $R$, since bonds in the beadspring model experience narrow fluctuations. Concomitantly, a large number of narrow perforations is formed at the merged surface $\mathcal{S}(R)$ - reflecting narrow interstitials in the local packing of the monomers - and $\chi(R)$ sharply drops to a negative minimum. Consistently, the minimum is located at $R \approx 0.5$ for all systems. The number of perforations (each carrying a contribution -1 in Eq. 12) is $\mathcal{N}_{T} \sim 0.6 N$ in all cases, independently of the backbone length and the number of cross-linkers. By further increasing $R$, perforations shrink and vanish, or are transformed into cavities, removing their negative contributions to $\chi(R)$. For distances $R \sim 1.2$ most of the interstitials have vanished and, accordingly, $\chi(R)$ exhibits a steep increase from the minimum. The surface $\mathcal{S}(R)$ is expected to form a single spherical polyhedron, without perforations and cavities, for suficiently large $R$, reaching the limit $\chi=1$. Interestingly, $\chi(R)$ approaches this limit very smoothly. Indeed, for the largest radii accessed in the calculation, $R \sim 6, \chi(R)$ is positive but still smaller than one. Actually $\chi(R)<1$ for all the investigated values of $R$. This suggests that cavities are much less frequent than perforations in $\mathcal{S}(R)$, and that the ultimate smooth behavior of $\chi(R)$ reflects open topologies for the nanoparticles, which may contain just a few open loops. This is confirmed by visual inspection of the nanoparticle configurations (Fig. 3), and is consistent with the results for the distributions $P(s)$ in Figs. 5 and 11 .

\section{COMPARISON WITH EXPERIMENTS}

Fig. 13 illustrates SEC/MALLS traces corresponding to: i) the poly(methyl methacrylate) precursor containing $35 \mathrm{~mol} \%$ of reactive $\beta$-ketoester functional groups, ii) 


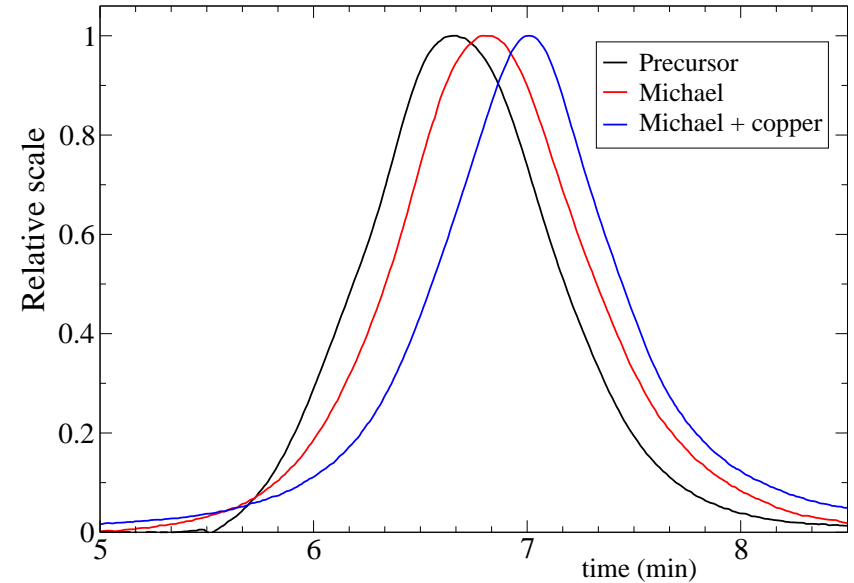

FIG. 13: SEC/MALLS traces of the poly(methyl methacrylate) precursor containing $35 \mathrm{~mol} \%$ of reactive $\beta$-ketoester functional groups (black), single-chain nanoparticles synthesized from this precursor exclusively via Michael addition reaction until completion (i.e., A cross-linked nanoparticles, red) and single-chain nanoparticles synthesized from this precursor via sequential intrachain Michael reaction plus metal complexation (i.e., A+B cross-linked nanoparticles, blue).

single-chain nanoparticles synthesized from this precursor exclusively via Michael addition reaction until completion (i.e., A cross-linked 'homofunctional' nanoparticles), and iii) single-chain nanoparticles synthesized from this precursor via sequential intrachain Michael reaction plus metal complexation (i.e., A+B cross-linked 'heterofunctional' nanoparticles). On going from the unlinked precursor to the cross-linked nanoparticles, a shift of the SEC/MALLS trace towards higher retention times is clearly observed in Fig. 13, which reflects a reduction in the hydrodynamic size of the resulting nano-objects (see below). The shift is larger for the cross-linked heterofunctional nanoparticles than for their homofunctional counterparts, in full consistency with the MD simulation results. Also, a decrease in the $M_{\mathrm{w}} / M_{\mathrm{n}}$ ratio is observed from 1.54 (precursor) to 1.42 (homofunctional nanoparticles) and to 1.38 (heterofunctional nanoparticles).

MALLS measurements allowed us to determine the average squared radii of gyration, $\left\langle R_{\mathrm{g}}^{2}\right\rangle$, of the precursor polymer, homofunctional and heterofunctional nanoparticles. As a representative example, precursor chains of $M_{\mathrm{w}}=800 \mathrm{kDa}$ showed a value of $\left\langle R_{\mathrm{g}}^{2}\right\rangle^{1 / 2}=25.7 \pm 0.1 \mathrm{~nm}$, whereas homofunctional and heterofunctional $(\mathrm{A}+\mathrm{B})$ nanoparticles synthesized from this precursor showed values of $\left\langle R_{\mathrm{g}}^{2}\right\rangle^{1 / 2}=19.6 \pm 0.3 \mathrm{~nm}$ and $\left\langle R_{\mathrm{g}}^{2}\right\rangle^{1 / 2}=10.6 \pm 0.5$ $\mathrm{nm}$, respectively. Similar results to the $\mathrm{A}+\mathrm{B}$ case were obtained for the $\mathrm{B}+\mathrm{A}$ cross-linked nanoparticles, i.e., by performing first the metal complexation process and second the intrachain Michael addition reaction.

Fig. 14 shows the SAXS results for the form factor [51] of the precursor and the homofunctional and heterofunctional nanoparticles. The large size of the macro-

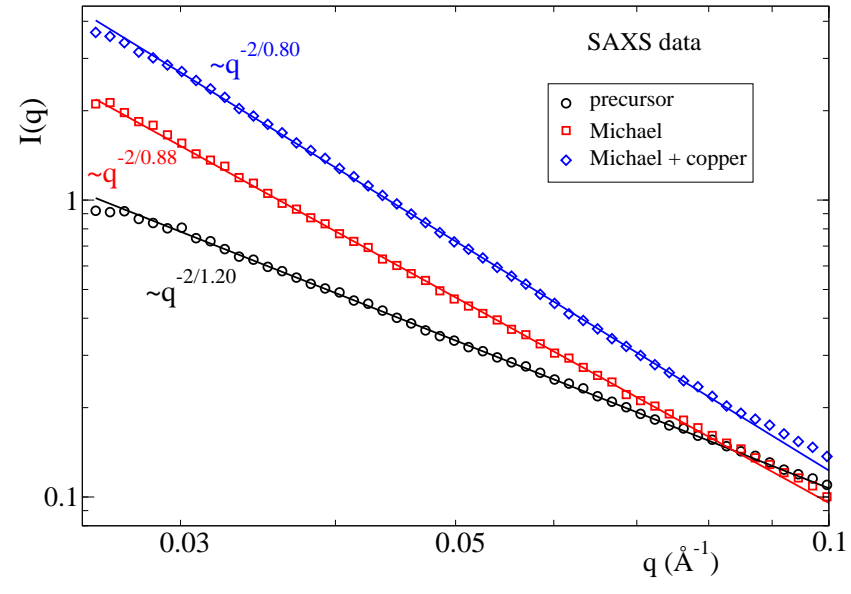

FIG. 14: Symbols: SAXS form factor for the unlinked precursor (circles) and for the nanoparticles obtained by Michael addition (squares), and by sequential Michael reaction and metal complexation (diamonds). Lines are fits to the power law $I(q) \sim q^{-2 / \nu}$. Exponents are indicated.

molecules assures the condition $q \gg 1 / R_{\mathrm{g}}$, and in the intermediate length regime accessed by these experiments the scattered intensity increases as a power law in $q$ determined by the Flory exponent $\nu$, as $I(q) \sim q^{-2 / \nu}$ (see, e.g., Ref. [51]). The precursor shows the expected behavior of a polymer chain in good solvent $(\nu=1.2)$. The form factors of the nanoparticles present markedly lower values of the $\nu$-exponent. These are even lower than in the case of random coils $(\nu=1)$, but still clearly above the exponent for globular objects $(\nu=0.67)$. Namely, the exponent decreases from $\nu=0.88$ for the homofunctional nanoparticles obtained by cross-linking mediated only by Michael addition, to $\nu=0.80$ for the heterofunctional ones obtained by sequential Michael reaction and metal complexation. We again find a qualitative agreement with the simulation results: the use of two crosslinking mechanisms leads to more compact nanoparticles.

The observed smaller values of the $\nu$-exponents in the real samples than in the MD simulations can be rationalized by the lower local flexibility of the first ones. Real polymers contain bending and torsional barriers that hinder local intramolecular deformations. These barriers are not present in the simulated flexible bead-spring model. We have made this choice for computational efficiency, since the presence of intramolecular barriers shift characteristic times to much longer time scales [60]. Thus, the lower deformability at the most local scales in the real precursor may facilitate chemical cross-linking at somewhat larger contour distances than in the bead-spring model, leading to somewhat more compact nanoparticles that are characterized by lower scaling exponents. Still, these are still clearly above those expected for globular objects, revealing that sparse topologies also dominate the conformations of the real single-chain soft nanoparticles. 


\section{CONCLUSIONS}

We have investigated, by means of MD simulations, the formation of soft nanoparticles by irreversible intramolecular cross-linking of homofunctional polymer precursors in good solvent conditions. Because of the initial selfavoiding character of the precursor, bonding at long contour distances, which is the efficient mechanism for global compactation, is a rare event that essentially occurs in the late stage of the cross-linking process. As a consequence, cross-linking may produce both compact and sparse soft nanoparticles even if the precursors have identical molecular weight and linker fraction. This has been confirmed by determining their scaling behavior and by a detailed quantitative analysis of their structural properties.

We have also studied the intramolecular cross-linking of heterofunctional polymers containing two types of orthogonal linkers. The resulting heterofunctional nanoparticles are, on average, smaller and more spherical than the homofunctional counterparts, though still a significant fraction of sparse objects is present. Further- more, simultaneous and sequential cross-linking of the two types of linkers lead to the same structural properties for the heterofunctional nanoparticles. The simulation results have been successfully compared with results from SEC/MALLS and SAXS experiments in real polymeric nanoparticles. The extensive and generic analysis presented in this article allows us to define more efficient experimental protocols for the synthesis of single-chain nanoparticles.

\section{ACKNOWLEDGEMENTS}

We acknowledge financial support from Projects No. MAT2012-31088 (Spanish Government) and No. IT654-13 (Basque Government). The X-ray measurements were performed with the SAXS instrument at the Centro de Física de Materiales (CFM) (CSIC$\mathrm{UPV} / \mathrm{EHU}$ ) acquired with the nominative subvention of the MEC to the CFM 2008. We acknowledge Centro de Supercomputación de Cataluña (CESCA, Spain) for generous allocation of CPU time.
[1] Ouchi, M.; Badi, N.; Lutz, J. F.; Sawamoto, M. Nature Chem. 2011, 3, 917-924.

[2] Sánchez-Sánchez, A.; Akbari, S.; Etxeberria, A.; Arbe, A.; Gasser, U.; Moreno, A. J.; Colmenero, J.; Pomposo, J. A. ACS Macro Lett. 2013, 2, 491-495.

[3] Sánchez-Sánchez, A.; Pérez-Baena, I.; Pomposo, J. A. Molecules 2013, 18, 3339-3355.

[4] Mackay, M. E.; Dao, T. T.; Tuteja, A.; Ho, D. L.; Van Horn, B.; Kim, H.-C.; Hawker, C. J. Nature Mater. 2003, 2, 762-766.

[5] Oria, L.; Aguado, R.; Pomposo, J. A.; Colmenero, J. Adv. Mater. 2010, 22, 3038-3041.

[6] Ruiz de Luzuriaga, A.; Pérez-Baena, I.; Montes, S.; Loinaz, I.; Odriozola, I.; García, I.; Pomposo, J. A. Macromol. Symp. 2010, 296, 303-310.

[7] Tuteja, A.; Duxbury, P. M.; Mackay, M. E. Macromolecules 2007, 40, 9427-9434.

[8] Whitaker, D. E.; Mahon, C. S.; Fulton, D. A. Angew. Chem. Int. Ed. 2013, 52, 956-959.

[9] Mecerreyes, D.; Lee, V.; Hawker, C. J.; Hedrick, J. L.; Wursch, A.; Volksen, W.; Magbitang, T.; Huang, E.; Miller, R. D. Adv. Mater. 2001, 13, 204-208.

[10] Harth, E.; Horn, B. V.; Lee, V. Y.; Germack, D. S.; Gonzales, C. P.; Miller, R. D.; Hawker, C. J. J. Am. Chem. Soc. 2002, 124, 8653-8660.

[11] Jiang, J.; Thayumanavan, S. Macromolecules 2005, 38, 5886-5891.

[12] Cherian, A. E.; Sun, F. C.; Sheiko, S. S.; Coates, G. W. J. Am. Chem. Soc. 2007, 129, 11350-11351.

[13] Croce, T. A.; Hamilton, S. K.; Chen, M. L.; Muchalski, H.; Harth, E. Macromolecules 2007, 40, 6028-6031.

[14] Adkins, C. T.; Muchalski, H.; Harth, E. Macromolecules 2009, 42, 5786-5792.

[15] Ruiz de Luzuriaga, A.; Ormategui, N.; Grande, H. J.; Odriozola, I.; Pomposo, J. A.; Loinaz, I. Macromol.
Rapid Commun. 2008, 29, 1156-1160.

[16] Ormategui, N.; Garcia, I.; Padro, D.; Cabanero, G.; Grande, H. J.; Loinaz, I. Soft Matter 2012, 8, 734-740.

[17] Radu, J. E. F.; Novak, L.; Hartmann, J. F.; Beheshti, N.; Kjoniksen, A.-L.; Nyström, B.; Borbély, J. Colloid Poly. Sci. 2008, 286, 365-376.

[18] Beck, J. B.; Killops, K. L.; Kang, T.; Sivanandan, K.; Bayles, A.; Mackay, M. E.; Wooley, K. L.; Hawker, C. J. Macromolecules 2009, 42, 5629-5635.

[19] Zhu, B.; Ma, J.; Li, Z.; Hou, J.; Cheng, X.; Qian, G.; Liu, P.; Hu, A. J. Mater. Chem. 2011, 21, 2679-2683.

[20] Zhu, B.; Qian, G.; Xiao, Y.; Deng, S.; Wang, M.; Hu, A. J. Polym. Sci., Part A: Polym. Chem. 2011, 49, 5330-5338.

[21] Jiang, X.; Pu, H.; Wang, P. Polymer 2011, 52, 3597 3602.

[22] Wang, P.; Pu, H.; Jin, M. J. Polym. Sci., Part A: Polym. Chem. 2011, 49, 5133-5141.

[23] Sánchez-Sánchez, A.; Asenjo-Sanz, I.; Buruaga, L.; Pomposo, J. A. Macromol. Rapid Commun. 2012, 33, $1262-1267$.

[24] Khanjani, P.; Pérez-Baena, I.; Buruaga, L.; Pomposo, J. A. Macromol. Symp. 2012, 321-322, 145-149.

[25] Dirlam, P. T.; Kim, H. J.; Arrington, K. J.; Chung, W. J.; Sahoo, R.; Hill, L. J.; Costanzo, P. J.; Theato, P.; Char, K.; Pyun, J. Polym. Chem. 2013, 4, 3765-3773.

[26] Pérez-Baena, I.; Barroso-Bujans, F.; Gasser, U.; Arbe, A.; Moreno, A. J.; Colmenero, J.; Pomposo, J. A. ACS Macro Lett. 2013, 2, 775-779.

[27] Seo, M.; Beck, B. J.; Paulusse, J. M. J.; Hawker, C. J.; Kim, S. Y. Macromolecules 2008, 41, 6413-6418.

[28] Foster, E. J.; Berda, E. B.; Meijer, E. W. J. Am. Chem. Soc. 2009, 131, 6964-6966.

[29] He, J.; Tremblay, L.; Lacelle, S.; Zhao, Y. Soft Matter 
2011, 7, 2380-2386.

[30] Appel, E. A.; Dyson, J.; del Barrio, J.; Walsh, Z.; Scherman, O. A. Angew. Chem. Int. Ed. 2012, 51, 41854189.

[31] Akagi, T.; Piyapakorn, P.; Akashi, M. Langmuir 2012, 28, 5249-5256.

[32] Mes, T.; van der Weegen, R.; Palmans, A. R. A.; Meijer, E. W. Angew. Chem. Int. Ed. 2011, 50, 5085-5089.

[33] Mavila, S.; Diesendruck, C. E.; Linde, S.; Amir, L.; Shikler, R.; Lemcoff, N. G. Angew. Chem. Int. Ed. 2013, 52, 5767-5770.

[34] Murray, B. S.; Fulton, D. A. Macromolecules 2011, 44, $7242-7252$.

[35] Tuten, B. T.; Chao, D.; Lyon, C. K.; Berda, E. B. Polym. Chem. 2012, 3, 3068-3071.

[36] Gillissen, M. A. J.; Terashima, T.; Meijer, E. W.; Palmans, A. R. A.; Voets, I. K. Macromolecules 2013, 46, 4120-4125.

[37] Hosono, N.; Gillissen, M. A. J.; Li, Y.; Sheiko, S. S.; Palmans, A. R. A.; Meijer, E. W. J. Am. Chem. Soc. 2012, 135, 501-510.

[38] Sánchez-Sánchez, A.; Akbari, S.; Moreno, A. J.; Lo Verso, F.; Arbe, A.; Colmenero, J.; Pomposo, J. A. Macromol. Rapid Commun. 2013, 34, -.

[39] Chao, D.; Jia, X.; Tuten, B.; Wang, C.; Berda, E. B. Chem. Commun. 2013, 49, 4178-4180.

[40] Liu, J. W.; Mackay, M. E.; Duxbury, P. M. EPL 2008, $84,46001$.

[41] Liu, J. W.; Mackay, M. E.; Duxbury, P. M. Macromolecules 2009, 42, 8534-8542.

[42] Ferrante, F.; Lo Celso, F.; Duca, D. Colloid Polym. Sci. 2012, 290, 1443-1450.

[43] Mather, B. D.; Viswanathan, K.; Miller, K. M.; Long, T. E. Prog. Polym. Sci. 2006, 31, 487-531.

[44] Kremer, K.; Grest, G. S. J. Chem. Phys. 1990, 92, 5057.
[45] Allen, M. P.; Tildesley, D. J. Computer Simulation of Liquids; Oxford University Press, Oxford, UK: 1989.

[46] Izaguirre, J. A.; Catarello, D. P.; Wozniak, J. M.; Skeel, R. D. J. Chem. Phys. 2001, 114, 2090.

[47] Smith, W.; Forester, T. R.; Todorov, I. T. The DL_POLY_2 User Manual, Version 2.19; STFC Daresbury Laboratory Daresbury, UK: 2009.

[48] Rottach, D. R.; Curro, J. G.; Budzien, J.; Grest, G. S.; Svaneborg, C.; Everaers, R. Macromolecules 2006, 39, 5521-5530.

[49] Frenkel, D.; Smit, B. Understanding Molecular Simulation; Academic Press, San Diego: 1996.

[50] Doi, M.; Edwards, S. F. The Theory of Polymer Dynamics; Oxford University Press, Oxford, UK: 1986.

[51] Rubinstein, M.; Colby, R. H. Polymer Physics; Oxford University Press, Oxford, UK: 2003.

[52] Rudnick, J.; Gaspari, G. Science 1987, 237, 384-389.

[53] Rawdon, E. J.; Kern, J. C.; Piatek, M.; Plunkett, P.; Stasiak, A.; Millett, K. C. Macromolecules 2008, 41, 8281-8287.

[54] Capone, B.; Coluzza, I.; Lo Verso, F.; Likos, C. N.; Blaak, R. Phys. Rev. Lett. 2012, 109, 238301.

[55] Lo Verso, F.; Panagiotopoulos, A. Z.; Likos, C. N. Phys. Rev. E 2009, 79, 010401.

[56] Koch, C.; Likos, C. N.; Panagiotopoulos, A. Z.; Lo Verso, F. Mol. Phys. 2011, 109, 3049-3060.

[57] Likos, C. N.; Mecke, K. R.; Wagner, H. J. Chem. Phys. 1995, 102, 9350.

[58] Hoffmann, N.; Ebert, F.; Likos, C. N.; Löwen, H.; Maret, G. Phys. Rev. Lett. 2006, 97, 078301.

[59] Hoffmann, N.; Likos, C. N.; Löwen, H. J. Phys.: Condens. Matt. 2006, 18, 10193.

[60] Bernabei, M.; Moreno, A. J.; Colmenero, J. Phys. Rev. Lett. 2008, 101, 255701. 